\title{
ARTICLES
}

\section{REVIEW OF THE ARTICLE ON “CARING FOR OLDER ADULTS WITH MENTAL HEALTH DISORDERS DURING THE PANDEMIC" AND ROLE OF A PSYCHIATRIC NURSE PRACTITIONER IN A COMMUNITY}

\author{
Ms. Ibha Sedenu* | Ms. Neena Mathew** \\ ${ }^{*} D N P, A P R N, F N P-B C, P H M N P-B C$ \\ ${ }^{*}$ MSN, FNP-BC, PMHNP-BC \\ DOI: http://doi.org/10.47211/trr.2021.v07i02.009
}

\begin{abstract}
This article 'Caring for the Older Adults with Mental Health Disorders during the Pandemic' is informative and accurate in its suggestions on caring for the elderly with mental illness. I find it particularly interesting how the simplest things such as spending time, engaging, and connecting to our elderly loved ones cause such a major impact on their mental health. As a clinician, these are lessons to rely on for effective communication and treating the client holistically. During the past few years of the Covid-19 pandemic, I have witnessed an emphasis on the elderly population due to their vulnerability to Covid-19. Often mentioned is physical health, but mental health is overlooked. I appreciate this article for its insights on mental health disparities among the elderly, and how health care providers can help.
\end{abstract}

Key words: Corona Virus Disease (COVID-19), psychological responses, geographical locations.

\section{ABOUT AUTHORS:}

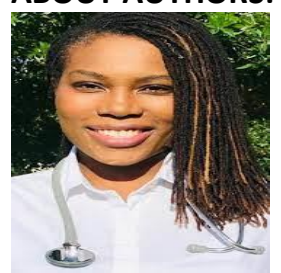

Dr. Ibha Sedenu is a dually Board Certified Family Nurse Practitioner and Psychiatric Mental Health Nurse Practitioner. She earned her Master of Science in Nursing from the University of Central Florida, a post-graduate board certificate in Psychiatry from Regis College-Massachusetts, and a Doctor of Nursing Practice from Touro University-Nevada. Her clinical experiences include assignments at Advent Health, Orlando Health, several central Florida Skilled Nursing facilities, and Behavioral Health facilities. As an Adjunct Professor at Regis College- Nursing Department, she also enjoys sharing her wealth of knowledge. Dr. Sedenu provides comprehensive evaluations, personalized medication management, therapeutic cognitive strategies, and specialist referrals as needed; to address the needs and desired outcomes of each individual. Dr. Ibha Sedenu brings compassion, enthusiasm, and a wealth of experience to improve your quality of life.

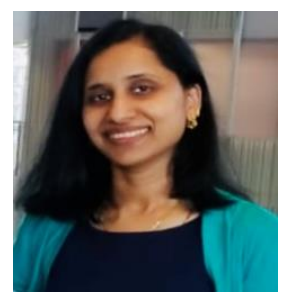

Ms. Mathew is a Board Certified Nurse Practitioner who is qualified to practice in both primary care and psychiatry; diagnose, treat, and prescribe medication for across the life span including children, adolescents, adults and older adults. She is currently working as a Lead Nurse Practitioner with Park Place Behavioral Healthcare, since 2016, which is a community mental health care facility in Orlando Florida. Some of her practice specialties include (but not limited to) ADHD, Depression, Anxiety and Bipolar. Mathew's style of practice can be traditional (prescribing up to date psychiatric medications) or alternative medicine (natural treatments including but not limited to vitamins, supplements, lifestyle modification, nutrition, exercise and stress management). She believes in the mind/body/spiritual connection, and we are made up as a whole person and not a series of parts, just as Hippocrates "The Father of Medicine." 


\section{ARTICLES}

\section{ROLE OF A PSYCHIATRIC NURSE PRACTITIONER}

Psychiatric Nurse Practitioners enjoy an exciting career, as every day can bring new patients, cases, and treatments to explore. For example, one day you may be counseling someone struggling with depression. The next day you might be running tests and trying to diagnose a deep-seated stress disorder. All the while, you will be making a difference by helping those with mental health issues. Patients with mental health disorders feel stigma and shame for their struggles, but your everyday commitment to their recovery is an incredible undertaking.

Some of daily tasks of a Psychiatric Nurse Practitioner include:

- Diagnosing patients for mental disorders, as well as being able to differentiate if a patient is experiencing a psychological or physical-based disorder

- Documenting a patient's past history and evaluating how that may have led to a mental disorder, such as family genetic history or past trauma

- Laying out a mental health treatment plan for recovery, including taking medication and attending counseling

- Prescribing medication and making sure a patient keeps up with their daily medication schedule

- Monitoring the effectiveness of a patient's treatment plan

- Collaborating with a patient's health team to potentially modify their care plan

- Educating families and patients on how to provide care at home

- Teaching mental health themed classes in topics such as stress reduction

- Meeting with your patients' treatment team to discuss developments

- Leading therapy sessions for individuals, families, or groups

\section{Contributions of Psychiatric Nurse Practitioner to the Society}

The combination of chronic illnesses associated with mental conditions cost the United States more than \$300 billion and another $\$ 210$ billion due to the work absenteeism costs attributed to employees' impairment (Kittelsrud, 2016). For instance, Reeves et al. (2011) established that in South Dakota, about three days each month were used for treating mental illness including depression, which results in loss of work hours. Remmers et al. (2009) revealed that medication adherence in chronic mental condition is poor and it escalates rehospitalization as well as worsens patient outcomes. According to the National Institute of Mental Health, nearly one in five adults in the U.S. lives with a mental illness - in 2016, that meant 44.7 million people who require proper medical attention. Providing this type of care is what a psychiatric nurse practitioner does on a daily basis. Equipped with an in-depth education and professional skills, psychiatric nurse practitioners give patients the treatment and guidance they need throughout their journey to wellness. Nurse practitioners working in the psychiatric mental health specialty assist individuals and their families and communities with a full range of psychiatric services. As they evaluate their patients' progress throughout their lifespan, psychiatric nurse practitioners provide support and education to families and communities. They can also be instrumental in policy development and health care reform as it relates to mental health issues and the treatment of mental health patients. Nurse practitioners are continuing to take on more varied roles in health care. Within the mental health field, psychiatric nurse practitioners perform a wide range of duties that help patients cope with mental health issues. Well-educated and experienced nurse practitioners have the necessary tools to make positive advances in the mental health of their patients, while promoting mental health awareness among individuals and communities across the nation.

According to the National Institute of Mental Health, nearly one in five adults in the U.S. lives with a mental illness in 2016, that meant 44.7 million people who require proper medical attention. Providing this type of care is what a psychiatric nurse practitioner does on a daily basis. Equipped with an in-depth education and professional skills, psychiatric nurse practitioners give patients the treatment and guidance they need throughout their journey to wellness.

The Job Responsibilities of a Psychiatric Nurse Practitioner

Nurse practitioners working in the psychiatric mental health specialty assist individuals and their families and communities with a full range of psychiatric services.

Psychiatric mental health nurse practitioners (PMHNP) are responsible for assessing the mental health status of patients by studying their relevant medical history, performing comprehensive psychiatric evaluations, and identifying risk factors that might affect a patient's mental health. They make diagnoses of their patients' mental illness based on their assessment, and then determine what will be the most effective plan of care. By utilizing 


\section{ARTICLES}

their knowledge of psychotherapy modalities and psychopharmacology, and in collaboration with doctors and other professionals, these advanced-practice nurses put their patients on a regimen of therapy and prescription medication to improve their mental health.

As they evaluate their patients' progress throughout their lifespan, psychiatric nurse practitioners provide support and education to families and communities. They can also be instrumental in policy development and health care reform as it relates to mental health issues and the treatment of mental health patients.

\section{Key Skills and Attributes}

The work that psychiatric nurse practitioners do is both emotionally and mentally demanding. It requires a broad set of professional skills honed over the course of their careers that allow these nurse practitioners to manage their stress and workload while providing a high-level of care.

\section{Professionalism}

The subject of mental health can be difficult for patients and their families to openly discuss. It is important that psychiatric nurse practitioners act professionally, showing respect and tact when communicating with patients and their families, and when assessing and evaluating a patient's mental health. They need to establish trust that will enable people to confide in them while maintaining organizational values and acting as an advocate for those coping with mental illness. In addition, psychiatric nurse practitioners must be able to manage the pressure and stress that comes with their position.

\section{Analytical Skills}

Determining the scope and specifics of a mental illness can be difficult because its signs are not often clear and may differ from patient to patient. Psychiatric nurse practitioners must be able to synthesize diverse data and patient research in conjunction with their in-depth medical knowledge and the insights from other professionals. The ability to analyze all of this information will allow them to design plans for care and therapy for their patients.

\section{Problem Solving}

Psychiatric nurse practitioners find solutions. It is their job to help patients to manage their mental health issues. Problem-solving skills are necessary throughout the treatment process. These skills help in identifying key risk factors, such as medical and family history of mental illness, trauma, substance abuse, and environment, among other factors. Psychiatric nurse practitioners must be able to collaborate with other professionals, using logic and reason when addressing emotional topics. They are also responsible for the continual evaluation of a patient's treatment plan and for finding alternate solutions as new challenges arise.

\section{CONCLUSIONS}

Nurse practitioners are continuing to take on more varied roles in health care. Within the mental health field, psychiatric nurse practitioners perform a wide range of duties that help patients cope with mental health issues. Well-educated and experienced nurse practitioners have the necessary tools to make positive advances in the mental health of their patients, while promoting mental health awareness among individuals and communities across the nation.

The future holds great promise and impact for psychiatric mental health nurse practitioners (PMHNP). We have the potential to improve the health and well-being of a future generation and improve public mental health. Yet, doing so requires a shift in ideology from the delivery of specialty based care to a primary care model - one based in nursing values and informed by the public health approach. Making this ideological shift does not require changes to the PMHNP standard of practice or scope, but does require the PMHNP to engage in collaborative relationships and to integrate many basic and advanced competencies. Expanded scope of practice can likely also encourage this re-visioning of the role. Educators, students, clinicians from other disciplines, policy-makers, and even children and families must advocate for change. The first step for our profession is to obtain more and better data about the demand for services and the current and projected workforce, as well as population-specific data (i.e. geriatric, child and adolescent, women's mental health). The profession must also seriously review the level of training needed in specialty populations in order to provide appropriate care. Training models, including the master's and clinical doctorate level preparation and formalized post-graduation options, must seek to address this provider gap as well as make efforts towards increasing the number of nurse practitioners able to practice. By challenging the current status quo of child and adolescent mental health care delivery, PMHNPs can be true leaders in the field and deeply impact the state of mental health 


\section{ARTICLES}

\section{REFERENCES}

1. Collins C., Hewson D.L., Munger R., Wade T. Milbank Memorial Fund; New York, NY: 2010. Evolving Models of Behavioral Health Integration in Primary Care. [Google Scholar]

2. Blackmore M.A., Carleton K.E., Ricketts S.M. Comparison of collaborative care and colocation treatment for patients with clinically significant depression symptoms in primary care. Psychiatr Serv. 2018;69(11):1184-1187. doi: 10.1176/appi.ps.201700569. [PubMed] [CrossRef] [Google Scholar]

3. Soltis-Jarrett V. The TANDEM3-PC: the foundation for an innovative, integrated behavioral health NP-led model of practice in rural primary care. Arch Psychiatr Nurs. 2019;33(1):2-10. doi: 10.1016/j.apnu.2018.08.007. [PubMed] [CrossRef] [Google Scholar]

4. Emerson M.R. Implementing a hybrid-collaborative care model: practical considerations for nurse practitioners. Issues Ment Health Nurs. 2019;40(2):112-117. doi: 10.1080/01612840.2018.1524533. [PubMed] [CrossRef] [Google Scholar]

5. University of Washington Principles of collaborative care. AIMS Center. https://aims.uw.edu/collaborative-care/principles-collaborative-care Published 2020. Published 2020. Accessed June 12, 2020.

6. Archer J., Bower P., Gilbody S. Collaborative care for depression and anxiety problems. Cochrane Database Syst Rev. 2012;10:CD006525. doi: 10.1002/14651858.CD006525.pub2. [PubMed] [CrossRef] [Google Scholar]

7. Atlantis E., Fahey P., Foster J. Collaborative care for comorbid depression and diabetes: a systematic review and meta-analysis. BMJ Open. 2014;4(4) doi: 10.1136/bmjopen-2013-004706. [PMC free article] [PubMed] [CrossRef] [Google Scholar]

8. Stewart J.C., Perkins A.J., Callahan C.M. Effect of collaborative care for depression on risk of cardiovascular events: data from the IMPACT randomized controlled trial. Psychosom Med. 2014;76(1):29-37. doi: 10.1097/PSY.0000000000000022. [PMC free article] [PubMed] [CrossRef] [Google Scholar]

9. Sowa N.A., Jeng P., Bauer A.M. Psychiatric case review and treatment intensification in collaborative care management for depression in primary care. Psychiatr Serv. 2018;69(5):549-554. doi: 10.1176/appi.ps.201700243. [PMC free article] [PubMed] [CrossRef] [Google Scholar]

10. Delaney K.R., Naegle M.A., Valentine N.M., Antai-Otong D., Groh C.J., Brennaman L. The effective use of psychiatric mental health nurses in integrated care: policy implications for increasing quality and access to care. J Behav Health Serv Res. 2018;45(2):300-309. doi: 10.1007/s11414-017-9555-x. [PubMed] [CrossRef] [Google Scholar]. 